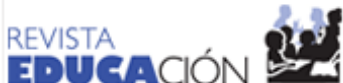

Revista Educación

ISSN: 0379-7082

ISSN: 2215-2644

revedu@gmail.com

Universidad de Costa Rica

Costa Rica

\section{Percepción docente sobre el ciberplagio universitario a través de un grupo de discusión por videoconferencia}

Gallent Torres, Cinta; Tello Fons, Isabel

Percepción docente sobre el ciberplagio universitario a través de un grupo de discusión por videoconferencia Revista Educación, vol. 45, núm. 2, 2021

Universidad de Costa Rica, Costa Rica

Disponible en: https://www.redalyc.org/articulo.oa?id=44066178039

DOl: https://doi.org/10.15517/revedu.v45i1.43527

\section{(c) $(1) \Theta$}

Esta obra está bajo una Licencia Creative Commons Atribución-NoComercial-SinDerivar 3.0 Internacional. 


\title{
Percepción docente sobre el ciberplagio universitario a través de un grupo de discusión por videoconferencia
}

\author{
University Faculty Perception of Cyber-Plagiarism: A Virtual Discussion Group \\ Cinta Gallent Torres \\ Universidad Internacional de Valencia, Valencia, España \\ cinta.gallent@campusviu.es \\ DOI: https://doi.org/10.15517/revedu.v45i1.43527 \\ Redalyc: https://www.redalyc.org/articulo.oa? \\ $\mathrm{id}=44066178039$
}

(iD https://orcid.org/0000-0002-4260-7594

Isabel Tello Fons

Universidad Politécnica de Cartagena, Centro

Universitario de la Defensa, España

isabel.tello@cud.upct.es

(iD https://orcid.org/0000-0001-7533-1064

Recepción: 30 Agosto 2020

Aprobación: 07 Enero 2021

\section{Resumen:}

Este estudio analiza el ciberplagio académico desde la perspectiva del profesorado del Grado en Traducción e Interpretación de la Universidad Internacional de Valencia. Dado que se trata de una práctica fraudulenta cada vez más extendida en las aulas universitarias, se hace necesario que el cuerpo docente reflexione sobre ella para evitar que se convierta en un recurso estructural por parte del alumnado en este nivel educativo. El estudio apuesta por una metodología cualitativa y, en concreto, el grupo de discusión, el cual se lleva a cabo por videoconferencia gracias a la participación de su claustro $(n=16)$. Como instrumento se diseña un guion de seis preguntas a partir de las cuales se pretende conocer (i) qué medidas adopta el profesorado para atajar estas conductas en el marco de la enseñanza virtual; (ii) qué responsabilidad le corresponde asumir cuando detecta un caso de plagio, y (iii) quién debe cuantificar la gravedad de dicho acto. Asimismo, se analiza la implicación de la institución en la lucha contra el ciberplagio y el tipo de apoyo que su profesorado requiere para hacer frente a estas prácticas. También se establece un protocolo de actuación de referencia, con la intención de sistematizar procedimientos y unificar criterios entre el personal docente de este grado. Para proceder a la recogida de datos, se decide grabar la sesión en la plataforma Blackboard Collaborate Ultra, de manera que la transcripción del material recopilado (tanto a nivel oral como escrito a través del chat de la sala) permita sintetizar la información en categorías codificables para su posterior análisis e interpretación. Entre las principales conclusiones cabe destacar que el profesorado percibe como injusto que la responsabilidad en la gestión de esta problemática recaiga sobre las personas docentes, por lo que requieren mayor apoyo institucional. Apuestan por contrarrestar este fenómeno mediante acciones que alerten al estudiantado de su gravedad e insisten en la necesidad de dirigir los esfuerzos de la comunidad académica hacia la sensibilización y concienciación de esta problemática.

Palabras Clave: Deshonestidad académica, Integridad académica, Propiedad intelectual, Enseñanza universitaria, Investigación cualitativa.

\section{ABstraCt:}

$=16$

This study explores academic cyberplagiarism from the perspective of undergraduate instructors for the Translation and Interpretation major at the International University of Valencia, Spain. Since cyberplagiarism has become increasingly widespread by university students, instructors must reflect on ways to deter students from using this fraudulent practice as a structural and educational resource. A qualitative methodology was used based on a virtual discussion group among participating faculty $(\mathrm{n}=16)$ who responded to a script that consisted of six questions designed to learn (i) how instructors tackle cyberplagiarism through a virtual teaching framework; (ii) their role and responsibility upon encountering a case of plagiarism among their students, and (iii) who is responsible for quantifying the gravity of these actions. The study also considers actions taken by the institution and the support it provides its faculty to address this practice and also established a guideline to standardized criteria among faculty members. The discussion was recorded on the Blackboard Collaborate Ultra and transcribed for purposes of data collection (the transcription included both the discussion and the chatbox comments). This facilitated classification of the collected material for 
subsequent analysis and interpretation. As a conclusion, it should be noted that the participating instructors generally expressed that they should not be held accountable for addressing cyberplagiarism and required broader institutional support to that end. They also expressed their commitment to counteract this problem and insisted that the academic community make a greater effort to raise awareness among students about the severity and consequences of such academic misconduct.

KEYWORDs: Academic Dishonesty, Academic Integrity, Intellectual Property, Higher Education, Qualitative Research.

\section{INTRODUCCIÓN}

Numerosas son las investigaciones que analizan el fenómeno de la deshonestidad académica, su gravedad y prevalencia en el marco de los estudios universitarios (Sureda-Negre, Cerdá-Navarro, Calvo-Sastre y ComasForgas, 2020); investigaciones que, desde enfoques distintos (descriptivos, empíricos y mixtos) y colectivos diferentes (profesorado, alumnado y centro), pretenden dar respuesta a una problemática cada vez más frecuente en la sociedad actual, pero para la que, por el momento, no existe una solución clara y evidente (Bergadaà, 2020).

Quizá la razón sea la falta de consenso por parte de la comunidad académica en la implementación de mecanismos que ayuden a detectar, reducir y contrarrestar las conductas fraudulentas en el ámbito universitario; o la falta de legislación que regule y penalice con severidad todo tipo de actuación inadmisible. A esto se añade la ausencia de una definición común sobre lo que se entiende por deshonestidad académica y las acciones que se identificarían como fraudulentas en este contexto, así como las consecuencias que se derivarían de ellas en el proceso de enseñanza-aprendizaje universitario.

Ciertamente, las acciones fraudulentas en las que incurre el alumnado universitario son muchas y muy variadas, pero, de entre todas ellas, la que mayor interés ha generado en la literatura especializada es la del plagio o ciberplagio (también conocido como plagio digital .cibernético). Según Fernández (2017), el plagio refiere a "la copia o la inclusión de ideas o fragmentos de otras obras en un trabajo propio sin incluir los créditos correspondientes a la fuente original" (p. 253), por tanto, sin reconocer la voz del verdadero autor. Cuando se utilizan medios electrónicos para apropiarse del pensamiento ajeno, esta acción pasa a denominarse ciberplagio. En esencia, su significado es el mismo que el de plagio, con la diferencia de que incluye en su definición un elemento adicional: el aspecto tecnológico; es decir, la alusión al medio utilizado para acceder y utilizar las ideas de un tercero de forma deshonesta. Se trata de una práctica que se realiza con el apoyo de las nuevas tecnologías y que fomenta lo que Casasola (2015) denomina como sabiduría virtual, una sabiduría falsa, aparente e irreal; un mundo en el que la información se manipula con la intención de conseguir un beneficio propio, en lugar de utilizarse para generar un nuevo conocimiento.

En la actualidad, dicha práctica, ya sea en forma de plagio o ciberplagio, requiere ser analizada desde distintas perspectivas. Si bien existen numerosas investigaciones que la analizan desde la perspectiva del alumnado (Childers y Bruton, 2016; Gallent y Tello, 2017, 2018, 2021; Cebrián-Robles, Raposo-Rivas, Cebrián de la Serna, Sarmiento-Campos, 2018a; Ochoa y Cueva, 2016; López-Gil y Fernández, 2019), resulta relevante centrarse ahora en el profesorado como agente dinamizador del proceso de enseñanzaaprendizaje. Interesa su perspectiva porque se hace necesario identificar aquellos elementos que, según este colectivo, incitan al alumnado a realizar un uso éticamente incorrecto de la información que consultan. También urge plantear propuestas y medidas que fortalezcan los procesos formativos a estos niveles. En este sentido, se tomará como punto de partida los estudios de Bruton y Childers (2016), Gourlay y Deane (2016) o Gottardello, Pàmies y Valverde (2017), quienes analizan este fenómeno desde la perspectiva docente e insisten en la falta de un marco conceptual que defina, delimite y tipifique las conductas fraudulentas, así como la gravedad de ellas. Además, apelan a la necesidad de establecer políticas claras que penalicen estas conductas, para evitar que la autoridad de quienes deciden penalizarlas o regularlas, a través de protocolos universitarios, se vea desacreditada. 
Este estudio se enmarca bajo este prisma, ya que pretende analizar el ciberplagio académico en el marco de la enseñanza virtual y desde la perspectiva del profesorado del Grado en Traducción e Interpretación de la Universidad Internacional de Valencia (España). Particularmente, esta investigación busca (i) identificar las representaciones del profesorado sobre el concepto de ciberplagio; (ii) compartir qué medidas o mecanismos adoptan para atajar estas conductas fraudulentas; (iii) analizar la responsabilidad que le corresponde asumir cuando detecta estos comportamientos ilícitos, y (iv) establecer un protocolo de actuación de referencia que permita sistematizar procedimientos y unificar criterios entre los docentes de este grado.

Los resultados y conclusiones alcanzados evidencian un primer paso hacia la reflexión colectiva sobre un problema de naturaleza ética que no solo afecta al ámbito académico, sino también a otras esferas de la sociedad.

\section{EL PROFESORADO: PIEZA CLAVE EN LA LUCHA CONTRA EL CIBERPLAGIO}

El profesorado, junto con el alumnado, es protagonista del proceso de enseñanza-aprendizaje que se desarrolla en un aula; como tal, debe adoptar una postura ejemplar ante conductas que, de manera voluntaria o accidental, sean inadmisibles. En este sentido, numerosos son los estudios que analizan el rol del profesorado ante esta problemática y coinciden en que "muchos profesores-investigadores tienden a tratar con indulgencia los casos [de plagio] que detectan" (Simonnot, 2014, p. 223); que existe un clima de silencio y complicidad ante la denuncia de estas prácticas (Luke y Kearins, 2012), y que se requiere formación docente en esta materia, ya que esto ayudaría a unificar criterios entre el claustro de cada centro.

En ocasiones, la indulgencia con la que se tratan estos casos parte del desconocimiento del alcance del fenómeno, de la falta de información respecto a las herramientas que fomentan la picaresca del estudiantado (Smodin.me, Photomath, Resoomer, Brainly, Resumidor LK, etc.) o de la falta de estrategias metodológicas sobre creación de tareas, elaboración de trabajos académicos o referencia de fuentes bibliográficas. En esta línea, autores como Comas y Sureda (2008) inciden en el tipo de tareas que se encomiendan al alumnado: actividades que se repiten año tras año, sin seguimiento alguno, en las que no se delimita el tema específicamente o no se evidencia el verdadero sentido por el cual se solicitan; actividades que carecen de conexión con la futura práctica profesional del estudiantado, que son desmotivadoras y mecánicas, y cuya realización no supone aprendizaje alguno.

En coherencia con lo anterior, los estudios que hacen referencia al rediseño de las tareas para mitigar el ciberplagio inciden en la importancia de fomentar la motivación y la ilusión al solicitarlas (López, 2014), en incitar al estudiantado a que piense de forma crítica (Heckler, Forde y Bryan, 2013) y en alentar la originalidad y el valor de las ideas propias (Gómez-Espinosa, Francisco y Moreno-Ger, 2016). Pero, por otra parte, el profesorado debe retroalimentar al alumnado debidamente, leer con profundidad sus trabajos y aportar una crítica constructiva que le sirva para mejorar.

Estudios como el de Sureda, Comas y Morey (2009), dirigidos a las personas docentes, concluyen que la profundidad con la que estas leen los trabajos del alumnado no es suficiente y reconocen su parte de culpa como un elemento importante en la comisión de plagio académico. Si bien la responsabilidad última de la comisión del acto es del alumnado, inevitablemente algunos de los factores que les motivan a plagiar implican al profesorado. Como ejemplo, entre las causas que motivan el ciberplagio, citadas por distintas personas autoras (Devlin y Gray, 2007; Ronda-Pérez, Seguí-Crespo, Tauste, Martínez y EsteveFaubel, 2015), se repiten cuestiones que tienen que ver con la relación, atención o seguimiento que el profesorado hace del alumnado. De hecho, el estudiantado indica que "la falta de acompañamiento que [el profesorado] brinda en los procesos de escritura académica y metodologías [...] no fomentan la construcción de conocimiento" (Ochoa y Cueva, 2016, p. 25), hecho que podría explicarse como una razón más de la adopción de conductas plagiarias. De nada sirve, pues, que se soliciten tareas al alumnado si el profesorado 
no le ofrece el apoyo y la retroalimentación adecuada para que siga aprendiendo de manera significativa, ya que es precisamente durante este proceso de acompañamiento en el que encuentra sentido a su formación.

Asimismo, estudios como el de Simonnot (2014) o Šprajc, Urh, Jerebi, Trivan y Jereb (2017) resaltan la desmotivación del alumnado como un factor fuertemente relacionado con el papel del profesorado en el desempeño de su labor docente. Por tanto, la manera en la que este dinamice el proceso de enseñanzaaprendizaje y potencie las capacidades, habilidades y destrezas del alumnado, repercutirá en el éxito de la formación.

Los estudios anteriores son valiosos por poner sobre la mesa algunas de las claves para la prevención del ciberplagio, en concreto, la mayor implicación del profesorado en las tareas que encomiendan a sus estudiantes y en el seguimiento que deben hacer de su aprendizaje.

Sin duda, la posición del profesorado es clave y delicada. Unida a una carencia formativa sobre el fenómeno, detectar una conducta fraudulenta implicaría enfrentarse a diversas diatribas morales. En primer lugar, puede desencadenar un sentimiento de desconfianza sobre el alumnado, lo cual supondría un perjuicio para quienes no copian. Asimismo, al denunciar un caso de plagio y comunicarlo al estudiantado o al centro, se puede encontrar con un protocolo institucional poco claro (aunque sí lo sea sobre el papel) o implicar el descrédito de la universidad en la que se colabora, lo cual lleva al cuerpo docente a reflexionar sobre la conveniencia de hacerlo público. Por otra parte, el mismo cuerpo docente pudo haber cometido plagio en el pasado, lo que le llevaría a afrontar esta práctica de forma más laxa o, todo lo contrario, de forma implacable. Si bien ninguno de los extremos sería conveniente, se requeriría del análisis pormenorizado de cada caso.

Otro asunto que pone en jaque al profesorado es el uso de programas antiplagio u otras herramientas digitales gratuitas que están accesibles en Internet. Gran parte del alumnado lleva ventaja a sus docentes respecto al uso de las herramientas tecnológicas y, por tanto, su pericia es superior a la hora de hacer trampas. Utilizar adecuadamente los programas antiplagio o conocer las herramientas que facilitan la comisión de conductas fraudulentas conlleva recibir una formación que no todo el profesorado universitario ha adquirido.

Ciertamente, los pasos que se deben seguir en la búsqueda de soluciones son bien sabidos y giran en torno a palabras clave, como formación y responsabilidad por parte de los agentes implicados. En este sentido, existe un amplio acuerdo entre las y los especialistas en que la vía para minimizar estas conductas pasa por la educación y la visibilización de la deshonestidad académica a través de cursos o seminarios dirigidos al profesorado y el alumnado (Lindahl y Grace, 2018; Cebrián-Robles, Rasposo-Rivas, Duarte-Freitas, 2018b); una formación que idealmente debería iniciarse en etapas educativas anteriores a la universidad y a la que deberían estar acostumbrados tanto el cuerpo docente como el alumnado. En cualquier caso, integrar dicha formación como una materia más del currículum, a pesar de su retraso, supone un paso adelante en la normalización y sensibilización del fenómeno dentro del sistema educativo (Lowe, Londino-Smolar, Wendeln y Sturek, 2018). Del mismo modo, el profesorado debería integrar un contenido específico, en sus respectivas materias, destinado a formar a su alumnado en la correcta citación de fuentes, la escritura académica o el uso de programas de gestión bibliográfica como Mendeley, Zotero . EndNote, lo que evidenciaría una acción consensuada por parte del colectivo docente.

Todos estos esfuerzos no tendrán sentido si el profesorado no es consciente del problema y decide ser indulgente a la hora de denunciar los casos de fraude. La responsabilidad del profesorado tiene un peso tan importante como la que se puede atribuir al alumnado, puesto que, si estos son los últimos responsables de los actos fraudulentos, los primeros juegan un papel decisivo en la prevención, concienciación y corrección del ciberplagio. En última instancia, a falta de una ética antiplagio que guíe al profesorado, las universidades deberían encargarse de identificar a la parte del profesorado que se implica poco en la lucha de estas prácticas, provocar la creación de espacios de reflexión sobre esta problemática, y dotar a la comunidad universitaria de los recursos, herramientas y formación necesaria para combatir el ciberplagio.

Sin duda, el profesorado es una pieza clave en el engranaje académico y en la lucha contra este fenómeno. Tal y como muestra la Figura 1, se encuentra en una posición intermedia entre el colectivo discente y el centro; 
una posición complicada, a la par que privilegiada, que le exige, por una parte, poner en marcha mecanismos de control, denuncia e información al alumnado sobre el alcance de estas prácticas y, por otra, reclamar al centro medidas de concienciación, sensibilización y formación que ayuden a contrarrestarlas.

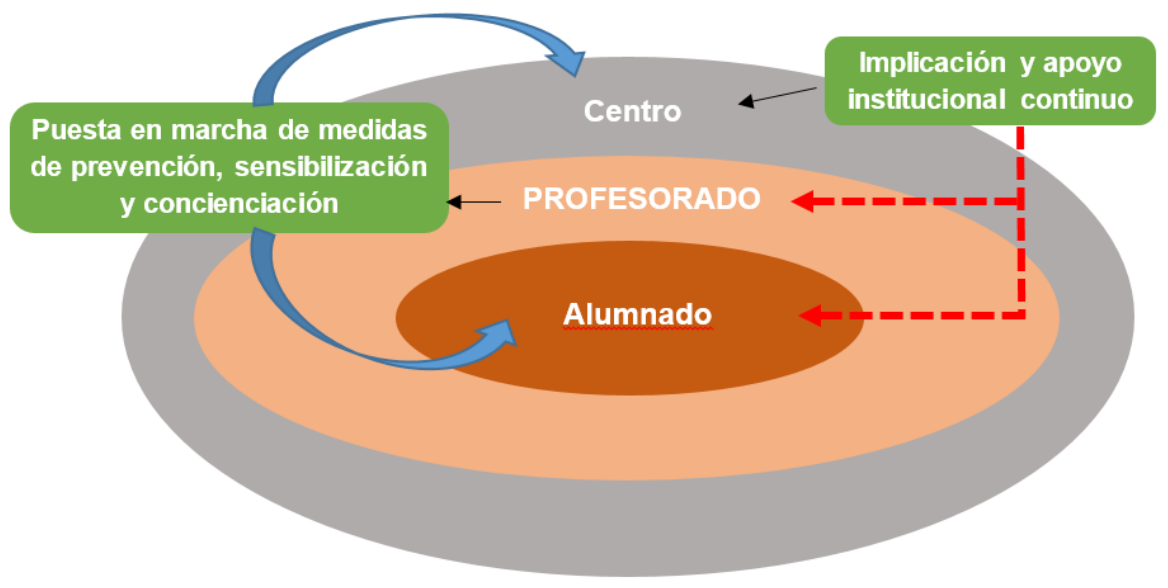

FIGURA 1.

Posición del colectivo docente en la comunidad académica Fuente: elaboración propia.

\section{ASPECTOS METODOLÓGICOS: DISEÑO E IMPLEMENTACIÓN DEL GRUPO DE DISCUSIÓN}

Este estudio se enmarca dentro de un proyecto de investigación más amplio que consta de dos fases: (i) el diseño de una encuesta ad hoc dirigida al profesorado del grado, y (ii) un grupo de discusión organizado con el mismo claustro en diciembre de 2019. Dado que los resultados de la encuesta se encuentran en proceso de análisis en estos momentos, el interés de este artículo se centra en compartir las conclusiones alcanzadas en la segunda fase del proyecto, es decir, en el grupo de discusión. Cabe indicar que se optó por esta técnica de recogida de información, como complemento de la encuesta, por presentar una serie de potencialidades que otros instrumentos de investigación no ofrecían. Especialmente se valoró que el método brindara la posibilidad de comprender percepciones colectivas, actitudes y sentimientos que serían inaccesibles sin la interacción del grupo, y que el uso de distintos códigos -silencios, registros, tonos de voz, gestos, etc. - fuera un estímulo para la creación de sentidos y significados (López, 2010). Además, mediante su aplicación no se pretendía llegar a un consenso sobre la temática planteada, sino buscar un sentido compartido por las personas participantes (Arboleda, 2008) dentro de una situación comunicativa relajada y espontánea.

En realidad, el grupo de discusión es una técnica de investigación de corte cualitativo, ampliamente validada por la comunidad científica (Callejo, 2001; Galeano, 2004; Sánchez y Murillo, 2010) que, si bien hace algunas décadas se utilizaba casi exclusivamente en estudios de mercado, poco a poco ha ido cobrando un especial interés y notoriedad en el ámbito académico (Infesta, Vicente y Cohen, 2012).

Lo interesante de esta metodología es que "la información surge en el propio escenario grupal donde las personas participantes se sienten estimulados por la presencia de los otros hacia quienes dirigen su intervención" (Infesta et al., 2012, p. 241). Dicha información forma parte de un proceso discursivo abierto, en el que entran en juego opiniones, actitudes y comportamientos propios de un colectivo en un contexto de interacción delimitado en el tiempo y en el espacio. Se hace referencia con ello a que el grupo de discusión que se conforma "no preexiste, ni continúa en el tiempo, sino que, una vez finalizado el encuentro, su permanencia no tiene razón de ser” (Sánchez y Murillo, 2010, p. 174). A pesar de ello, es una técnica que permite recoger una cantidad de datos nada desdeñable para cualquier investigación. 
De acuerdo con lo anterior, en este caso concreto, para el diseño del grupo de discusión se tuvo en consideración los objetivos teóricos, metodológicos y epistemológicos de la investigación, así como las recomendaciones de algunas personas especialistas en esta materia (Canales y Peinado, 1998; Callejo, 2001; Galeano, 2004), en relación con la duración de la sesión, el espacio o el tamaño de la muestra. Se apostó por criterios más flexibles y menos directivos, que atendieran al colectivo participante y a la modalidad de enseñanza virtual de la universidad. Es importante remarcar esta información porque, a partir de la búsqueda bibliográfica realizada y el análisis del corpus documental existente sobre esta metodología, no se localizó ninguna experiencia cualitativa similar llevada a cabo a través de grupos de discusión por videoconferencia, hecho que aporta valor a la investigación.

En este sentido, y dado que la universidad imparte clases en un entorno online, sería incoherente, al igual que poco factible, haber realizado un grupo de discusión que se desarrollara presencialmente. De ahí que el medio en el cual se desarrolle el grupo de discusión cobre un especial protagonismo en este tipo de dinámicas.

En esta investigación, el grupo de discusión -basado en las opiniones, ideas y sugerencias expresadas por las personas docentes en primera persona sobre su experiencia en esta materia- se realizó a través de una sala virtual a la que se accede por videoconferencia mediante la herramienta Blackboard Collaborate Ultra. Si bien la elección de dicho espacio vino condicionada por la modalidad de enseñanza de la universidad, su utilización fue, sin duda, un acierto. Afortunadamente, hoy en día, los medios tecnológicos favorecen el desarrollo de estos métodos de investigación, les confieren mayor precisión y fiabilidad a los estudios y permiten la grabación de las sesiones para su posterior visualización (tal y como ocurrió en este estudio). Dicha grabación de voz e imagen era impensable hace unos años, ya que el registro de los diálogos se llevaba a cabo mediante grabador-casete (Callejo, 2001), cinta sonora (Arboleda, 2008) o grabadora de voz digital (López, 2010).

Sin embargo, la videoconferencia ofrece un valor añadido a la persona investigadora, quien, además de analizar el contenido textual de las ideas planteadas, puede realizar un importante trabajo de observación, análisis e interpretación de la imagen, el lenguaje no verbal, el tono de voz, los turnos de intervención e, incluso, los silencios. Si hace tan solo unos años se consideraba que la grabación por vídeo era más intrusiva que la de audio (López, 2010) y que condicionaba la respuesta de las personas que intervienen porque les generaba incomodidad, esta idea va perdiendo peso gracias a las ventajas que ofrecen los medios tecnológicos actuales para seguir profesionalizando estas metodologías en el ámbito educativo.

Por lo que respecta al grupo de profesorado colaborador, de 29 que imparten materias en este grado, únicamente 16 participaron en esta segunda fase; todas y todos contestaron la encuesta online. Esto fue debido a la dificultad que supuso encontrar una fecha y hora que se adaptara a sus agendas profesionales, ya que no todos residen en España. En cualquier caso, el tamaño de la muestra fue el idóneo para que las personas informantes pudieran compartir sus percepciones y experiencias y, al mismo tiempo, hubiera diversidad de opiniones. De acuerdo con Sánchez y Murillo (2010), “el tamaño de los grupos suele oscilar desde tres o cuatro participantes hasta un máximo de doce o trece” (p. 184); si esto se extrapola a un contexto online, el número de participantes puede ser un poco mayor, siempre y cuando se respeten los objetivos de la investigación, sus intervenciones sean pertinentes y sus discursos aporten información al estudio (Arboleda, 2008).

Por otra parte, algunas personas autoras recomiendan que los actantes no se conozcan entre sí (Arboleda, 2008; Sánchez y Murillo, 2010), hecho inevitable en este caso, ya que pertenecen al mismo ámbito profesional y colaboran en el mismo título universitario, es decir, en el Grado en Traducción e Interpretación de la Universidad Internacional de Valencia. No obstante, sí se respetaron los criterios de homogeneidad intragrupo y heterogeneidad intergrupal propuestos por varias personas autoras (Sánchez y Murillo, 2010; Infesta et al., 2012), es decir, que el grupo en cuestión compartía características comunes (es parte del profesorado del grado y especialista en el ámbito de la traducción e interpretación), pero al mismo tiempo, 
aportaba perspectivas distintas (desarrolla ocupaciones diferentes en otros centros educativos), diversidad profesional que fomenta la variedad de opiniones.

Otro aspecto importante que se debe destacar en el diseño del grupo de discusión es el guion de preguntas que se utiliza durante la reunión, cuyo contenido y estructura no debe condicionar la dinámica de grupo ni limitar la espontaneidad de las intervenciones (Canales y Peinado, 1998), sino generar un discurso libre, mediante el cual se expliquen y construyan significados. De acuerdo con la duración de la sesión (120 minutos), el guion se articuló en torno a las siguientes cuestiones:

- ¿Qué responsabilidad corresponde asumir al profesorado que detecta un caso de plagio? ¿Quién debería calificar y cuantificar su gravedad?

- ¿A qué se debe la disparidad de criterios docentes para gestionar cada caso?

- ¿Por qué los casos de plagio identificados suelen solucionarse de manera individual entre el profesorado y el alumnado y apenas trascienden a la dirección de título?

- ¿Qué cambios metodológicos deberían implementarse (tanto en las clases y las actividades como en los exámenes) para evitar estas conductas?

- Dado que no existe un procedimiento institucional específico ante un caso de plagio, ¿qué procedimiento sugeriría el profesorado implantar en este grado?

- ¿Cómo podría implicarse más a la institución en la lucha contra este fenómeno?

A pesar de ser un guion que sugiere seguir una dinámica de pregunta-respuesta, en realidad, pretende promover la interacción grupal y abordar el fenómeno del ciberplagio desde diferentes perspectivas. Para ello, la figura moderadora será una pieza clave en la dinamización del grupo, ya que dirigirá la discusión y velará por la consecución de los objetivos de la investigación.

\section{DESARROLLO DE LA INVESTIGACIÓN}

El desarrollo de la investigación giró en torno a cinco fases que fueron configurándose conforme avanzaba el estudio (ver Figura 2):

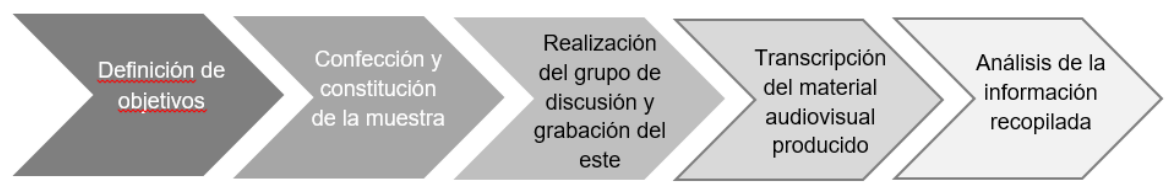

FIGURA 2.

Fases de la investigación

Fuente: elaboración propia.

A continuación, se describe el protocolo de trabajo que se siguió en cada una de ellas.

En la primera fase del estudio se delimitaron y definieron los objetivos de la investigación para que fueran lo más claros posible para todas las personas participantes. Dichos objetivos se abordaron desde cuatro ejes principales: el conocimiento del término ciberplagio y su alcance en el marco de la enseñanza virtual; las distintas medidas (preventivas, de sensibilización y concienciación) adoptadas por parte del profesorado para evitar conductas plagiarias; la responsabilidad que corresponde asumir al personal docente que detecta un caso de plagio, y el establecimiento de un protocolo de actuación de referencia para que el profesorado de este grado pueda implementarlo en futuras ediciones. Esta fase se complementó con una revisión bibliográfica sobre metodología cualitativa y aplicación de grupos de discusión a investigaciones de esta naturaleza y la creación de un primer borrador del guion estructurado de la sesión. 
La segunda fase de la investigación consistió en la selección de participantes y la constitución de la muestra. Por el tipo de estudio, la selección fue intencional dado que se limitó a un grupo de docentes muy concreto: el claustro del Grado en Traducción e Interpretación $(n=29)$. Del claustro participante en el grupo de discusión $(n=16)$, tres fueron hombres y trece mujeres, de 39 años como media. En el grupo había docentes que impartían asignaturas de lengua, traducción, documentación e interpretación en todos los cursos del grado, hecho que permitió obtener una amplia variedad de ideas y perspectivas.

En esta fase también se revisó el guion inicial y se implementaron algunas mejoras, como la reducción del número de preguntas, la eliminación de algunas cuestiones ya incluidas en la encuesta y la selección de algunos detonadores que se lanzan al grupo para su posterior discusión. La versión final del guion incluyó seis preguntas generales, a partir de las cuales surgieron otros subtemas que fueron también analizados.

La tercera fase del estudio se centró en el desarrollo del grupo de discusión. Se invitó a las personas informantes a participar voluntariamente en el proyecto y conectarse a la sesión a través de la sala de coordinación del grado. Cada participante disponía de cámara y micrófono para intervenir; dado que la calidad de sonido y de audio era óptima, el acto se desarrolló con total normalidad. Además, al ser una videoconferencia grabada, el equipo de investigación pudo acceder a ella y visualizarla en numerosas ocasiones para realizar el análisis y la interpretación de los datos.

Durante la cuarta fase del proyecto, se procedió a la transcripción del material producido, con el objetivo de realizar una segmentación temática por unidades de significado y sintetizar los datos en categorías codificables (Suárez, 2005). De esta manera, la información se convierte en abarcable y su posterior análisis resulta más sencillo. El material textual generado a partir de la transcripción facilitó dicho análisis, aportó una visión general del estudio y gran cantidad de información sobre las representaciones del claustro respecto al fenómeno del ciberplagio.

Finalmente, la quinta fase se refiere al análisis e interpretación de la información recopilada según el contexto y participantes. Dado que se trata de una sesión de videoconferencia grabada, el análisis se realizó a partir del examen de cada una de las manifestaciones que se produjeron en el grupo (tanto las intervenciones orales como las escritas a través del chat de la sala), con la intención de encontrar concurrencias o singularidades relacionadas con el objeto de estudio, tal y como apuntan Sánchez y Murillo (2010). El resultado del análisis permitió agrupar las cuestiones inicialmente planteadas en cuatro categorías, que dieron respuesta a cada uno de los objetivos del estudio.

\section{Resultados}

Los resultados obtenidos a partir del análisis del grupo de discusión dieron respuesta a las preguntas de investigación que se plantearon a las personas participantes, las cuales se agruparon en torno a las siguientes categorías: (i) responsabilidad ante un caso de ciberplagio; (ii) propuesta de protocolo a seguir tras su detección; (iii) medidas docentes para evitar futuros plagios; e (iv) implicación del centro en la lucha contra esta práctica fraudulenta.

Antes de presentar los resultados obtenidos, cabe indicar que el grupo de discusión inició con la contextualización del estudio y los objetivos de la sesión. Seguidamente, se compartieron algunas definiciones de los términos plagio y ciberplagio aportadas por el profesorado en la fase de la encuesta ad hoc; definiciones que evidenciaron el acuerdo existente en cuanto a su significado y alcance, y que sirvieron de punto de partida para la discusión. El claustro coincidió en que esta práctica alude a la copia de contenidos de terceros online y offine, y uso de los estos como sifueran propios; en que se trata de una apropiación indebida de material intelectual ajeno, sea en forma impresa o digital; y en que el plagio como tal se identifica con cualquier trabajo desarrollado en el que se copian materiales de otra persona autora sin mencionarla explícitamente o haciéndolos pasar por propios, mientras que el ciberplagio es lo mismo, pero recurriendo a los medios cibernéticos que facilita Internet, lo que multiplica de forma exponencial esa posibilidad. 
En este sentido, el profesorado tiene una idea acertada sobre lo que significa plagiar o ciberplagiar, pero todavía existen dudas sobre qué prácticas, más allá de la copia literal o mal reformulada de las ideas de un tercero, podrían también identificarse con una conducta plagiaria. Por ejemplo, la inclusión de referencias bibliográficas que sí existen, pero que no han sido utilizadas para la elaboración del trabajo entregado; la mención de la obra de un autor sin incluir los datos correctos para poder localizarla (año de publicación, número de página, etc.); la utilización de contenidos de trabajos entregados en cursos anteriores para reutilizarlos en las asignaturas del año en curso; la introducción una información traducida en un documento nuevo y adjudicada a su autoría; o la inclusión del nombre de algún compañero en algún trabajo o publicación sin que haya participado en la redacción del este, entre otras muchas acciones que recoge la literatura actual sobre esta temática (Cebrián-Robles et al., 2018b).

Fruto de las ideas compartidas, se alcanzan los siguientes resultados que se agrupan en torno a estas categorías:

\section{A) Responsabilidad ante un caso de ciberplagio}

Ante la pregunta sobre qué responsabilidad le corresponde asumir al profesorado que detecta un caso de plagio y quién debe cuantificar su gravedad, el equipo docente considera que no pueden ser los responsables finales de la irresponsabilidad de sus estudiantes. Intencionalmente o no, el alumnado es quien comete una acción fraudulenta y, por tanto, quien debe atenerse a las consecuencias. Si bien el profesorado detecta en primera instancia dicho fraude, el centro debería respaldarle con una normativa clara y tajante. Sin dicho apoyo, el profesorado se siente desprotegido y afronta estas acciones desde la individualidad. De hecho, cuando detecta un caso de ciberplagio, las medidas que toma son muy dispares: una parte del personal docente se limita a la simple reprimenda o la charla empática con el alumnado; otra opta por pedirle que rehaga la actividad copiada o suspenderle la convocatoria en curso; otra, sin embargo, es partidaria de aplicar medidas más severas, como la suspensión directa de la asignatura (en sus dos convocatorias) o la mención del acto cometido en el expediente académico del alumnado.

De ahí la importancia de que existan directrices institucionales bien definidas, que ayuden a la comunidad universitaria (tanto al profesorado como al estudiantado) a conocer qué se entiende por ciberplagio, cómo puede evitarse y qué penalizaciones conllevan estas conductas en el contexto académico. Dichas medidas no solo servirían de guía de actuación y de fuente de información y referencia para la comunidad universitaria, sino que también servirían para amedrentar a aquel alumnado que pudiera querer (ciber)plagiar, y dar la confianza suficiente al equipo docente a la hora de denunciar posibles casos de plagio. Al saberse firmemente amparados y amparadas por las instituciones, el número de docentes con actitud pasiva ante el problema descendería significativamente. Según Ochoa y Cueva (2014), el ciberplagio es "un fenómeno que involucra como responsables a profesores, estudiantes, programa curricular y sistema educativo, [aunque] con distinto peso funcional" (p. 97). El profesorado se muestra de acuerdo con esta afirmación e insiste en que la función del profesorado debería alejarse de la perspectiva sancionadora para abordarse desde una perspectiva ético-formativa. Ciertamente se debe alentar al estudiantado a hacer un uso responsable de las fuentes que consultan, a utilizar y hacer valer sus propias ideas, y a generar conocimiento a partir de la lectura de otras personas autoras. Pero la relación no debe verse dañada por sugerir o implementar medidas que velen por el respeto de la propiedad intelectual de un tercero o incluso la de las propias compañeras y compañeros.

Esta última idea es pertinente porque, en este grado en concreto, la mayoría de las asignaturas que se imparten son materias prácticas de traducción de especialidades distintas (literaria, científico-técnica, económico-administrativa, etc.). Al alumnado se le pide que traduzca textos reales en las distintas combinaciones lingüísticas, cuyas dificultades de traducción inevitablemente resuelven en conjunto vía WhatsApp, Skype o correo electrónico. Sin duda, es consciente de que ciberplagiar implica apropiarse de las ideas de las demás personas, pero cuando se trata de una traducción, el significado del término se disipa, ya que 
piensan que presentar la misma versión de traducción que un compañero es realizar un trabajo colectivo o se debe a una coincidencia entre las soluciones de traducción aportadas y no implica incurrir en plagio. En este caso, prevalece el sentimiento de compañerismo o la creencia de que es probable que dos o más estudiantes den con dos soluciones idénticas correctas frente al hecho de cometer una acción ilícita. Pero el no exigírsele que realice una aportación teórica, reformule una idea o incorpore una cita literal en una actividad de estas características, no le aleja de la posibilidad de cometerlo. Es más, cuando esto ocurre, no entiende por qué se les acusa de ello y su reacción suele ser poco positiva.

$\mathrm{Al}$ hilo de esto, múltiples y bien distintas son las reacciones del estudiantado tras comunicarle que ha cometido plagio, lo que produce que se desencadene una situación de incomodidad para el profesorado. Algunas personas integrantes del equipo docente opinan que, una vez detectado el fraude, la responsabilidad de su gestión debería externalizarse, bien a través de evaluadores externos, tribunales de docentes que impartan asignaturas similares o de la propia dirección del título, pero que el personal docente debería quedarse al margen del incidente. Por el contrario, otra parte de las personas participantes apuestan por informar directamente al estudiantado y aplicar las medidas sancionatorias necesarias sin miedo a posibles represalias. El estudiantado no puede gozar de impunidad ante este tipo de actos deshonestos y, para ello, tanto el profesorado como el centro deben actuar en consonancia. Si bien la parte responsable final del acto cometido es el alumnado, establecer límites a la actuación como docentes no es nada sencillo, sobre todo si la normativa interna de centro no especifica el protocolo en estos casos, no aclara las responsabilidades de cada colectivo ni informa al estudiantado de las consecuencias que estas acciones tienen para su futuro profesional.

\section{B) Propuesta de protocolo tras la detección de un caso de ciberplagio}

La necesidad de establecer un protocolo que marque las directrices de actuación ante esta problemática se debe a la disparidad de criterios entre el profesorado que detecta un caso de plagio; que estos casos no trascienden institucionalmente, porque apenas se reporten a la dirección de título se genera cierta desinformación sobre la frecuencia con la que se cometen; $y$, en paralelo, que el estudiantado no identifique estas prácticas deshonestas como una falta grave.

Aunque la normativa de la Universidad sobre plagio es clara, no es suficiente si no se acompaña de un procedimiento detallado sobre cómo actuar ante la nueva cultura de la copia, especialmente desde una perspectiva preventiva, de concienciación y sensibilización. En el caso de la Universidad Internacional de Valencia, el centro tipifica el plagio como una falta muy grave en el Reglamento de Régimen Disciplinario del Alumnado, aprobado por resolución rectoral el pasado 11 de febrero de 2019. La Universidad Internacional de Valencia (2019), en su artículo segundo, especifica las sanciones que se aplicarían si se incurre en el plagio:

...las faltas muy graves relativas a plagios y al uso de medios fraudulentos para superar las pruebas de evaluación tendrán como consecuencia la pérdida de la convocatoria correspondiente, así como el reflejo de la falta y su motivo en el expediente académico. (p. 5)

Más allá de esta mención en el reglamento, cuyo alcance es un tanto limitado, no se informa al profesorado (cuando se les contrata) de la existencia de un protocolo institucional sobre la manera de proceder ante estas conductas. De ahí el desarrollo de esta iniciativa. De la misma manera, el estudiantado tampoco está informado de la existencia de esta normativa. Si bien es pública y cualquier persona la puede consultar en el sitio web de la institución, no se publicita, se recuerda o se llama la atención sobre ella hasta que el profesorado, a título individual y de manera voluntaria, incluye alusiones en sus guías docentes o explican la normativa en sus clases.

En este sentido, el grupo de discusión fomentó el debate, consenso y esbozo de una primera propuesta de protocolo propia que permitiera sistematizar actuaciones, delimitar responsabilidades y concretar medidas y sanciones para evitar cualquier tentación antiacadémica en las aulas. 
En líneas generales, la propuesta de protocolo que se planteó fue la siguiente:

1. El profesorado sospecha que una tarea (actividad, trabajo, examen, etc.) está total o parcialmente copiada.

2. El profesorado confirma dicha sospecha al localizar la fuente primaria que sirvió de inspiración al estudiantado, bien a través de las herramientas antiplagio disponibles en la plataforma (Turnitino SafeAssign), otras aplicaciones de consulta gratuita en la red (Viper, PlagScan, TinEye, Copyleaks, PlagiarismDetect.org) o la inclusión literal del fragmento plagiado en un buscador genérico como Google, Yahoo o Bing. Por supuesto, el sentido común y la experiencia docente en esta materia son recursos muy válidos para identificar este tipo de conductas.

3. Realiza una comparación de ambas versiones a través de aplicaciones como Workshare Compare o Dupli Checker y elabora un breve informe.

4. Contacta con la Dirección de título para informar de dicho incidente.

5. La Dirección se asegura de que efectivamente la sospecha de la comisión del ciberplagio es cierta y la registra en un documento propio que recoge los casos de plagio reportados durante los últimos tres años.

6. La Dirección contacta de nuevo al profesorado y acuerdan la correspondiente penalización. Es entonces cuando la persona docente comunica este hecho al estudiantado (si el plagio se ha cometido en una actividad en grupo, todas las personas integrantes recibirán la misma penalización).

7. Por último, se realizará seguimiento de la respuesta, reacción y justificación por parte del estudiantado sobre el acto cometido.

Dado que la primera reacción del alumnado es bastante previsible, el profesorado deberá actuar con diplomacia, para conseguir que esta experiencia, a priori frustrante y delicada, se convierta en un capítulo formativo más en el proceso de aprendizaje del estudiantado. Así pues, se recomienda que el profesorado aproveche estas oportunidades para alentar al alumnado a utilizar responsablemente las ideas de las demás personas y, sobre todo, a desarrollar las suyas propias, ya sea en un trabajo de fin de grado, en una tarea de traducción o en una actividad de lengua o interpretación.

Este protocolo se irá profesionalizando con la experiencia, pero representa un excelente punto de partida para el cuerpo docente. Se pretende, pues, trabajar de manera coordinada y transparente, para que cuanta más información se disponga sobre estas conductas en este grado, más fácil resulte abordarlas. En este sentido, algunos profesores y profesoras también reclaman información sobre el estudiantado que plagió en los primeros cursos del grado o en asignaturas similares a las que imparten, pero no con la intención de crear una «lista negra» que catalogue un desafortunado error de citación en su día, sino con la finalidad de identificar las circunstancias en las que se cometieron, las asignaturas en las que lo hicieron, los motivos que utilizaron para justificarlo y las sanciones que se aplicaron en cada caso.

\section{C) Medidas docentes para evitar futuros casos de ciberplagio}

Si bien durante la discusión también se abordaron medidas de prevención o sensibilización ya propuestas por la literatura especializada, como los códigos de honor, las comisiones éticas o los distintos programas de software antiplagio (Nakandaraki, 2016; Fernández, 2017), el interés de las personas participantes se centró en compartir otras prácticas concretas, que ayudaran a mejorar la coordinación docente en materia de ciberplagio, entre las cuales se destacan las siguientes: 
- Advertir formalmente al alumnado, en las primeras sesiones del curso, de que sus trabajos van a ser evaluados y analizados con las herramientas antiplagio. Especial atención merecerán los trabajos de fin de grado.

- Comprometerse a explicar al alumnado cómo se redacta un trabajo académico, cómo se evalúan críticamente las fuentes bibliográficas y cómo estas deben citarse para evitar incurrir en plagio. El claustro valora la posibilidad de redactar una breve guía digital que resuma los aspectos más importantes sobre la elaboración de trabajos en el marco de la traducción e interpretación.

- Proponer tareas más creativas que motiven al estudiantado a documentarse y a dar su opinión crítica sin necesidad de recurrir sistemáticamente a Internet.

- Utilizar los informes generados mediante las herramientas antiplagio desde una perspectiva formativa, compartirlos con el estudiantado y conferirles la importancia relativa que merecen.

- Formar un tribunal o grupo de docentes del grado que adopten el rol para la evaluación cuando un miembro del claustro tenga la sospecha de que se ha cometido plagio en un trabajo, actividad o prueba, y necesite una segunda opinión.

- Incluir el ciberplagio como un aspecto recurrente a tratar en el orden del día de las reuniones de claustro, para que se sigan compartiendo experiencias docentes sobre estas prácticas.

- Recordar al alumnado la obligación de conocer el Reglamento de Régimen Disciplinario accesible desde la página web de la Universidad Internacional de Valencia (www.universidadviu.com/ normativa). Un buen momento para llevar a cabo esta acción, como se esboza más arriba, sería al inicio del curso, cuando el profesorado presenta sus materias al estudiantado.

- Implementar, a partir de la próxima edición, la propuesta de protocolo ad hoc e informar a la dirección de título de su funcionamiento o necesidad de mejora.

- Seguir actualizando el documento de registro de conductas fraudulentas para que, a corto plazo, aporte información sobre si las medidas planteadas tienen una repercusión positiva en el desarrollo formativo y ético del estudiantado.

- Seguir compartiendo experiencias y casos de plagio que se hayan identificado en las respectivas materias. Informalmente, el profesorado debe sentirse cómodo para contactar con el equipo de dirección de la titulación o con sus compañeros y compañeras, y compartir experiencias, inquietudes, pedir consejo, etc.

Tal y como un profesor dijo durante la sesión: quien no la hace, no la teme; esta actitud debería ser la dinámica general del estudiantado cuando elabora sus trabajos académicos, ya que a veces se olvida de que así es por culpa de unas pocas personas que utilizan la picaresca como atajo para conseguir buenos resultados.

\section{D) Implicación del centro en la lucha contra esta práctica fraudulenta}

La implicación del centro es fundamental para acelerar la puesta en marcha de medidas que el profesorado considera positivas en el marco de la enseñanza online. Por ejemplo, ante la dificultad que supone minimizar cualquier tentación ilícita en una prueba evaluativa en línea, la universidad toma una serie de medidas que dificultan que esto ocurra; medidas como la activación de un sistema de reconocimiento facial del alumnado, la grabación de la sesión del examen, el bloqueo de cualquier aplicación externa al campus durante la realización del este o la aceptación de una advertencia de seguridad y normativa.

No obstante, estas medidas podrían complementarse con otras que refuercen la seguridad de las pruebas y pusieran en valor el verdadero sentido del proceso de enseñanza-aprendizaje:

- La aceptación de una cláusula específica para cada asignatura, mediante la cual el estudiantado indique que todas sus aportaciones (escritas u orales) son originales y propias. 
- La firma de un documento, mediante el cual el estudiantado se compromete a no cometer plagio en el examen final e, incluso, a tener apagado el móvil durante este tiempo para evitar posibles conversaciones por WhatsApp o Telegram con otros compañeros o compañeras.

- La planificación de seminarios obligatorios sobre la utilización de fuentes digitales y ciberplagio para todo el estudiantado del grado.

- La incorporación, en los distintos planes de estudios, de una asignatura transversal sobre documentación, manejo de bases de datos, consulta de glosarios terminológicos, etc. (en este grado sí existe, pero debería también ofertarse en el resto de estudios).

- La creación de exámenes con contraseña y con un tiempo bien delimitado para su elaboración.

- La creación de exámenes en los que el alumnado desarrolle una idea y no conteste a un listado de preguntas tipo test.

- La imposibilidad de que se permitan entregas ilimitadas de cualquier actividad o prueba.

- La creación de materiales (vídeos, guías u otros documentos de consulta) sobre citación y referencias, accesibles para toda la comunidad universitaria.

- La creación de un espacio o sala virtual permanente dirigida al alumnado, para que pueda consultar o resolver una duda puntual sobre la utilización de fuentes bibliográficas o, incluso, solicitar una tutoría con docentes especializadas o especializados en la materia.

- El lanzamiento de campañas publicitarias y de sensibilización en las que participe tanto el profesorado como el alumnado.

Implicarse en la lucha contra el ciberplagio es tan fácil como impulsar iniciativas como esta, crear grupos de investigación que analicen este fenómeno desde distintas perspectivas, escuchar y compartir buenas prácticas con otras universidades españolas e, incluso, establecer colaboraciones con instituciones internacionales que cuenten con mayor experiencia en esta materia; porque incurrir en conductas deshonestas no es un hecho que afecte únicamente a una titulación, sino a toda una institución, una comunidad, una sociedad.

\section{Discusión, CONCLUSIONES Y LIMITACIONES}

Los resultados de este estudio han permitido cumplir con los objetivos planteados al inicio del trabajo y han puesto de manifiesto la percepción del cuerpo docente de un grado universitario oficial online sobre un tema importante en el marco académico. Ciertamente, los aspectos tratados arrojan datos de gran utilidad para esta investigación, así como para investigaciones futuras en esta materia.

Para retomar aquellas cuestiones más relevantes, cabe señalar que al preguntarles por la responsabilidad que debe recaer sobre este grupo en cuanto al ciberplagio, los profesores y las profesoras acuerdan que esta no debería recaer exclusivamente sobre el personal docente. Perciben como injusto que todo el peso de esta problemática dependa de ellos y ellas, de la manera en que la traten, de su denuncia, su gestión, resolución, etc. Necesitan sentir un respaldo de la institución, tanto por la Coordinación del Grado como por la Universidad. Este apoyo les ayudaría a afrontar los casos de fraude con más seguridad y tranquilidad, a sabiendas de que cuentan con la institución en la que colaboran.

Por otra parte, al tratarse el tema de confeccionar una propuesta de protocolo tras la detección de un caso de ciberplagio, el profesorado se decanta por un proceso sencillo y claro que pase por la comunicación con la Dirección del Grado, de tal manera que puedan aunarse puntos de vista y medidas punitivas entre ambas partes. De nuevo, se observa la importancia que se le otorga a contar con el apoyo institucional. El protocolo acordado, descrito anteriormente, es un reflejo de las necesidades de un claustro que muestra la actitud y la voluntad de contrarrestar el fraude académico, pero que a veces no cuenta con los medios burocráticos para hacerlo; se alejan del prototipo de docente que, si bien penaliza al alumnado que plagia, no indaga en las causas o propicia el diálogo con el estudiantado. El profesorado utiliza los programas antiplagio facilitados 
por la Universidad, así como otras herramientas accesibles en línea para cerciorarse de la comisión del plagio y poder dirigirse al alumnado con todos los datos. No obstante, sus esfuerzos se ven difuminados ante una reglamentación de centro inexistente o una normativa interna, cuyo cumplimiento no se ha exigido con contundencia.

En línea con lo anterior, cuando se le preguntó al profesorado por la implicación de la Universidad ante esta problemática, la opinión del claustro fue unánime en cuanto a los protocolos de actuación en caso de ciberplagio. Las personas docentes apuntaron la necesidad de disponer de unas pautas claras y eficaces, que sistematicen el procedimiento que debería seguirse tras una posible detección. A esta cuestión se añade la necesidad de sentir respaldo, especialmente por parte de la Universidad, que debería mantener una política visible en favor de la honestidad e integridad académica.

Otro aspecto en el que hay unanimidad es en poder recopilar un listado de casos de ciberplagio en el grado, lo que denota el interés por el tema y, al mismo tiempo, evidencia la necesidad de comprobar que otras personas docentes también detectan casos de fraude y se enfrentan a las mismas situaciones. El profesorado alega que contar con esta información le beneficiaría para tomar nota de las medidas adoptadas por sus colegas y seguir aunando criterios.

En relación a las medidas docentes para evitar futuros casos de ciberplagio, el claustro se decanta por los aspectos más prácticos de la docencia en el día a día. Se inclina unánimemente por aquellas acciones que alerten al estudiantado de la gravedad de cometer ciberplagio: manifestar la gravedad de esta práctica en clase, incluir alguna mención sobre ella en las guías docentes, concienciar al alumnado de la existencia del Reglamento de Régimen Disciplinario de la Universidad, elaborar una guía sobre la correcta redacción de un trabajo académico, etc., medidas que valoran también otros estudios sobre la misma temática (Gottardello et al., 2017). De todo ello se desprende la preocupación por el tema, la voluntad de querer evitar enfrentamientos innecesarios con posibles plagiarios y la necesidad de dirigir los esfuerzos hacia la sensibilización y concienciación de esta problemática, una idea en la que también coinciden otras personas autoras (Bergadaà, 2020; Cebrián, Raposo y Sarmiento, 2020).

En definitiva, si bien el grupo de discusión ha sido un instrumento óptimo para cumplir los objetivos del estudio, los resultados alcanzados se complementarán con los de la encuesta. Las preguntas planteadas en el grupo de discusión son distintas a las recogidas en esta, ya que se consideró oportuno que el claustro desarrollara abiertamente su punto de vista, sin limitarse a marcar una respuesta única.

Cabe mencionar que, dado a que se trata de la primera experiencia de grupo de discusión de forma online de la que se tiene constancia, no es posible comparar si los resultados obtenidos expuestos más arriba difieren de los que se obtuvieron en grupos de discusión presenciales realizados con anterioridad. Estas dinámicas tienen en común, no obstante, la aportación de matices y opiniones que los sujetos no podrían verter de otra forma (encuestas e incluso entrevistas individuales), pues el factor de debate, que plantea acertadamente el formato de grupo de discusión, alienta a las personas participantes a expresar sus opiniones de manera espontánea, al solidarizarse con sus colegas o, por el contrario, a discrepar en alguna idea compartida.

Otra de las limitaciones encontradas es el número de participantes, el cual es reducido porque así lo requiere esta técnica de investigación. En un futuro, sería interesante poder organizar otros grupos de discusión en paralelo, con docentes del mismo grado (incluso con quienes que no pudieron participar en esta ocasión), para comparar los resultados obtenidos. También podría proponerse la creación de un grupo de discusión que incluya a docentes de otras titulaciones, alumnado y profesorado de un mismo grupo y curso, o a docentes de otras universidades, incluso, de enseñanza secundaria. De la misma forma, sería interesante poder confrontar estudiantes, docentes y autoridades académicas de una misma universidad para tratar un tema como este. En definitiva, el grupo de discusión online permitiría reunir participantes de diferente procedencia con mayor facilidad y, por tanto, llegar a obtener resultados más rápidamente.

Por último, los resultados que arroja este análisis incitan a seguir explorando esta herramienta como técnica de investigación en futuros estudios científicos. 
Se considera, pues, que se han cumplido los objetivos planteados al inicio del estudio y se han aportado resultados cualitativos interesantes, que han permitido avanzar en la reflexión de un fenómeno sobre el cual se sigue teorizando mucho, pero cuyas medidas deben llevarse a la práctica de forma inminente.

\section{REFERENCIAS BIBLIOGRÁFICAS}

Arboleda, L. (2008). El grupo de discusión como aproximación metodológica en investigaciones cualitativas. Revista de la Facultad Nacional de Salud Pública, 26(1), 69-77. Recuperado de https://dialnet.unirioja.es/servlet/artic ulo?codigo $=5079739$

Bergadaà, M. (2020). Le temps. Entre science et création. Caen, Francia: EMS, Management \& Société.

Bruton, S. y Childers, D. (2016). The ethics and politics of policing plagiarism: a qualitative study of faculty views on student plagiarism and Turnitin. Assessment \& Evaluation in Higher Education, 41(2), 316-330. doi: https://d oi.org/10.1080/02602938.2015.1008981

Callejo, J. (2001). El grupo de discusión: introducción a una práctica de investigación. Barcelona: Ariel.

Canales, M. y Peinado, A. (1998). Grupos de discusión. En M. Delgado y J. Gutiérrez (Ed.) Métodos y técnicas cualitativas de investigación en Ciencias Sociales (pp. 287-316). Madrid: Síntesis.

Casasola, W. (2015). La sabiduría virtual: el ciberplagio universitario. Revista de Lenguas Modernas, 23, 429-433. doi: https://doi.org/10.15517/rlm.v0i23.22364

Cebrián-Robles, V., Rasposo-Rivas, M., Cebrián de la Serna, M. y Sarmiento-Campos, J.A. (2018a). Percepción sobre el plagio académico de estudiantes universitarios españoles. Educación XXI, 21(2), 105-129. doi: https://doi.o $\mathrm{rg} / 10.5944 /$ educXX1.20062

Cebrián-Robles, V., Raposo-Rivas, M. y Duarte-Freitas, M. C. (2018b). Acceso libre y antiplagio en los repositorios institucionales y bibliotecas de las Facultades de Educación en España, Relatec. Revista Latinoamericana de Tecnología Educativa, 17(2). Recuperado de http://dx.medra.org/10.17398/1695-288X.17.2.41

Cebriá, V., Raposo, M. y Sarmiento, J. (2020). Study of the reasons for and measures to avoid plagiarism in young students of Education. Profesorado. Revista de Curriculum y Formación del Profesorado, 24(1), 50-74. doi: http ://doi.org/10.30827/profesorado.v24i1.8572

Childers, D. y Bruton, S. (2016). Should it be considered plagiarism? Student perceptions of complex citation issues. Journal of Academic Ethics, 14(1), 1-17. doi: https://doi.org/10.1007/s10805-015-9250-6

Comas, R. y Sureda, J. (2008). El ciberplagi acadèmic: esbrinant-ne les causes per tal d'enllestir les solucions. Digithum, (10), 1-8. Recuperado de https://www.redalyc.org/pdf/550/55001005.pdf

Devlin, M. y Gray, K. (2007). In their own words: a qualitative study of the reasons Australian university students plagiarise. Higher education research and development, 26(2), 181-198. doi: https://doi.org/10.1080/0729436 0701310805

Fernández, A. (2017). Estrategias y herramientas tecnológicas para evitar el plagio académico. En E. Morales Campos (Coord.), La infodiversidad y el uso ético del conocimiento individual y colectivo (pp. 253-275). México: UNAM, Instituto de Investigaciones Bibliotecológicas y de la Información. Recuperado de http://eprints.rclis.org/313 26/1/Etica\%202017.pdf

Galeano, M. (2004). Estrategias de investigación social cualitativa: el giro en la mirada. Medellín: La Carreta.

Gallent, C. y Tello, I. (2017). Percepción del alumnado de traducción de la Universidad Internacional de Valencia (VIU) sobre el ciberplagio académico. Revista Digital de Investigación en Docencia Universitaria, 11(2), 90-117. doi: https://doi.org/10.19083/ridu.11.563

Gallent, C. y Tello, I. (2018). Le cyberplagiat dans le cadre de l'éducation supérieure espagnole : explorer les causes pour atteindre des solutions concrètes. Synergies Espagne, 11, 195-212. Recuperado de https://www.gerflint.fr /Base/Espagne11/gallent_tello.pdf 
Gallent, C. y Tello, I. (2021). L'intégrité dans l'enseignement supérieur espagnol : trois mondes parallèles. En M. Bergadaà y P. Peixoto (Dirs.). L'urgence de l'intégrité académique, (pp. 55-68). Caen: Éditions EMS. Questions de Société.

Gómez-Espinosa, M., Francisco, V. y Moreno-Ger, P. (2016). El impacto del diseño de actividades en el plagio de Internet en educación superior. Comunicar, 24(48), 39-48. doi: https://doi.org/10.3916/C48-2016-04

Gottardello, D., Pàmies, M. y Valverde, M. (2017). Professors' perceptions of university student's plagiarism: a literature review. BID: Textos universitaris de biblioteconomia i documentació, 39 (diciembre). doi: https://dx.d oi.org/10.1344/BiD2017.39.12

Gourlay, L. y Deane, J. (2016). Loss, responsibility, blame? Staff discourses of student plagiarism. Innovations in Education and Teaching International, 49(1), 19-29. doi: http://doi.org/10.1080/14703297.2012.647780

Heckler, N., Forde, D. y Bryan, C. (2013). Using writing assignment designs to mitigate plagiarism. Teaching Sociology, 41(1), 94-105, doi: https://doi.org/10.1177/0092055X12461471

Infesta, G., Vicente, A. y Cohen, I. (2012). Reflexiones en torno al trabajo con grupos de discusión en ciencias sociales. Intersticios. Revista sociológica de pensamiento critico, 6(1), 233-244. Recuperado de https://dialnet.unirioja.es/ servlet/articulo?codigo $=3828017$

Lindahl, J. y Grace, D. (2018). Students' and supervisors' knowledge and attitudes regarding plagiarism and referencing. Research Integrity and Peer Review, 3(10). doi: https://doi.org/10.1186/s41073-018-0054-2

López, I. (2010). El grupo de discusión como estrategia metodológica de investigación: aplicación a un caso. Edetania, 38, 147-156. Recuperado de https://revistas.ucv.es/index.php/Edetania/article/view/303

López-Gil, K. S. y Fernández, M. C. (2019). Representaciones sociales de estudiantes universitarios sobre el plagio en la escritura académica. Íkala. Revista de Lenguaje y Cultura, 24(1), 119-134. doi: https://doi.org/10.17533/ud ea.ikala.v24n01a06

López, J. (2014). Analyzing and reducing plagiarism at university. European Journal of Education and Psychology. 7(2),131-140. Recuperado de https://www.researchgate.net/publication/268813256_Analyzing_and_reducin g_plagiarism_at_university

Lowe, M. S, Londino-Smolar, G., Wendeln, K. E. A. y Sturek, D. L. (2018). Promoting academic integrigy through a stand-alone course in the learning management system. International Journal for Educational Integrity, 14(13). doi: https://doi.org/10.1007/s40979-018-0035-8

Luke, B., Kearins, K. (2012). Attribution of words versus attribution of responsibilities: Academic plagiarism and university practice. Organization, 19(6), 881-889. doi: https://doi.org/10.1177/1350508412448857

Nakandaraki, M. (2016). Plagio: ¿Qué es? ¿Qué hacer para evitarlo? Y ¿cuál es su implicancia científico-profesional? Revista Ciencia e Investigación Médico Estudiantil Latinoamericana (CIMEL), 21(1), 2-4. Recuperado de https ://www.cimel.felsocem.net/index.php/CIMEL/article/view/621

Ochoa, L. y Cueva, A. (2014). El plagio y su relación con los procesos de escritura académica. Forma y función, 27(2), 95-113. doi: https://doi.org/10.15446/fyf.v27n2.47667

Ochoa, L. y Cueva, A. (2016). Percepciones de estudiantes acerca del plagio: datos cualitativos. Revista Encuentros, 14(2), 25-41. doi: https://doi.org/10.15665/rev14i2.822

Ronda-Pérez, E., Seguí-Crespo, M., Tauste, A., Martínez, J. y Esteve-Faubel, J. (2015). Experiencia piloto de evaluación de una intervención para evitar el plagio académico en alumnos universitarios. Investigación y propuestas innovadoras de redes UA para la mejora docente. Universidad de Alicante. Recuperado de https://rua.ua.es/dsp ace/handle/10045/50652

Sánchez, M. y Murillo, P. (2010). Innovación educativa en España desde la perspectiva de grupos de discusión. Profesorado. Revista de Curriculum y Formación de Profesorado, 14(1),171-189. Recuperado de https://recyt.fe cyt.es/index.php/profesorado/article/view/42194

Šprajc, P., Urh, M., Jerebi, J., Trivan, D. y Jereb, E. (2017). Reasons for plagiarism in higher education. Organizacija, 50(1), 33-45. doi: https://doi.org/10.1515/orga-2017-0002

Simonnot, B. (2014). Le plagiat universitaire, seulement une question d'éthique ? Questions de communication, 26, 219-233. doi: https://doi.org/10.4000/questionsdecommunication.9304 
Cinta Gallent Torres, et al. Percepción docente sobre el ciberplagio universitario a través de un ...

Suárez, M. (2005). El grupo de discusión: una herramienta para la investigación cualitativa. Barcelona: Laertes.

Sureda, J., Comas, R. y Morey, M. (2009). Las causas del plagio académico entre el alumnado universitario según el profesorado. Revista Iberoamericana de Educación, 50,197-220. Recuperado de https://www.redalyc.org/pdf/8 00/80011741011.pdf

Sureda-Negre, J., Cerdá-Navarro, A., Calvo- Sastre, A. y Comas-Forgas, R. (2020). Las conductas fraudulentas del alumnado universitario español en las evaluaciones: valoración de su gravedad y propuestas de sanciones a partir de un panel de expertos. Revista de Investigación Educativa, 38(1), 201-219. doi: https://doi.org/10.6018/rie. 358781

Universidad Internacional de Valencia. (2019). Reglamento de Régimen Disciplinario de los alumnos. Recuperado de h ttps://www.universidadviu.com/download/file/17709

\section{INFORMACIÓN ADICIONAL}

Cómo citar: Gallent-Torres, C. y Tello-Fons, I. (2021). Percepción docente sobre el ciberplagio universitario a través de un grupo de discusión por videoconferencia. Revista Educación, 45(2). Recuperado de http://d oi.org/10.15517/revedu.v45i1.43527 